\title{
Restriction enzymes and their use in molecular biology: An overview
}

\author{
Francesca Di Felice ${ }^{1}$, Gioacchino Micheli ${ }^{2}$ and Giorgio Camilloni ${ }^{1,2} *_{\odot}$ \\ ${ }^{1}$ Dipartimento di Biologia e Biotecnologie, Sapienza, Università di Roma, Piazzale A. Moro 5, 00185 Roma, \\ Italy \\ ${ }^{2}$ Istituto di Biologia e Patologia Molecolari, CNR, Roma, Piazzale A. Moro 5, 00185 Roma, Italy \\ *Corresponding author (Email, giorgio.camilloni@uniroma1.it)
}

MS received 24 October 2018; accepted 3 January 2019; published online 8 April 2019

\begin{abstract}
Restriction enzymes have been identified in the early 1950s of the past century and have quickly become key players in the molecular biology of DNA. Forty years ago, the scientists whose pioneering work had explored the activity and sequence specificity of these enzymes, contributing to the definition of their enormous potential as tools for DNA characterization, mapping and manipulation, were awarded the Nobel Prize. In this short review, we celebrate the history of these enzymes in the light of their many different uses, as these proteins have accompanied the history of DNA for over 50 years representing active witnesses of major steps in the field.
\end{abstract}

Keywords. Cloning; DNA manipulation; history; restriction enzymes

\section{Introduction}

Historia magistra vitae: Cicero's expression, drawn from his almost 2000-year-old De Oratore, brilliantly synthesizes how the understanding of present reality must value past experience.

This requirement is highly relevant to scientific studies and is nicely exemplified by the references every scientific article relies on: new knowledge banks on previous acquisitions and acknowledges them. In this light, we present an overview on the experimental significance of a specific class of proteins, type II restriction enzymes. It has been more than half a century since the first studies on these molecules. Their fundamental role as tools for the characterization and manipulation of DNA quickly made them very well known within the scientific community. Highlighting their relevance to DNA studies goes in parallel with the history of the double helix and may appeal also to those who, while not working on DNA, are nonetheless interested in the progresses made by investigations in this area.

\section{Restriction and modification}

Major observations in microbial genetics revealing the capability of bacteria to resist bacteriophage infections were published in the early 1950s (Luria and Human 1952;
Bertani and Weigle 1953). These works describe the changes the infectious capacity of the phage undergoes during the growth cycle, showing that these alterations are not due to de novo mutations or selective processes. Subsequently, it was ascertained that the underlying defense system is based on DNA sequence-specific endonucleases, which restrict viral action (hence the term restriction enzymes) by digesting phage DNA, coupled to a corresponding DNA methyltransferase activity, which safeguards the integrity of host DNA by modifying potential cutting sites through the addition of methyl groups (reviewed in Wilson and Murray 1991).

For their seminal contribution to the discovery and use of restriction enzymes, Werner Arber, Dan Nathans and Hamilton Smith were awarded the 1978 Nobel Prize for Physiology and Medicine. Arber had hypothesized that these enzymes were able to bind DNA at sites represented by specific DNA sequences (Arber 1965). Smith had verified Arber's hypothesis by using purified enzymes and showing that they were able to cut symmetric and specific nucleotide sequences (Kelly and Smith 1970; Smith and Wilcox 1970). Nathans had realized the possible exploitation of these enzymes, producing the first restriction cutting site maps of specific DNA fragments (Danna and Nathans 1971). Since Arber's, Smith's and Nathans' studies, the characterization of restriction enzymes has developed very rapidly and four 
different enzyme classes have been identified. The enzymes belonging to class II are commonly exploited in manipulating and characterizing DNA. They only require $\mathrm{Mg}^{2+}$, and no ATP, to recognize their target DNA sequence and cleave it. Many restriction enzymes, originating from a wide range of bacterial species, belong to this class and hundreds of recognition/cutting sequences have been identified. Using different combinations of restriction enzymes, many different ways of characterizing and manipulating DNA have become possible (Williams 2003). Comparative analyses have shown that type II restriction enzymes are members of a large superfamily of proteins, called PD-(D/E)XK nucleases, characterized by a conserved core fold (a common $\alpha / \beta$ motif) but lacking significant sequence similarity except for critical residues of their active site (Knizewski et al. 2007). This poses interesting and as-yet open questions about the evolutionary pathways of the many members of this family (Gupta et al. 2012).

Class I, III and IV restriction enzymes are much less frequently employed in DNA studies because of their lower DNA-cutting specificity. Rather than coinciding with their recognition sequences, their cutting sites often localize far away and/or are not sequence specific. In addition, their restriction activity is not always separated from the methylation activity and their number is not as abundant as that of class II restriction endonucleases. Finally, the activity of class I, III and IV restriction enzymes is often ATP-dependent (reviewed in Roberts et al. 2003).

\section{Basic use of restriction enzymes: physical DNA mapping}

Since the first experiments by Danna and Nathans (1971), the major use of restriction enzymes was aimed at locating their cutting sites on selected DNA molecules. In an era where effective nucleotide sequence determination methods were yet under development, the specificity of the restriction cleavage profiles of different DNA regions allowed to easily compare them, resulting in an unprecedented flow of highly interesting structural, functional and evolutionary studies on many gene systems (Holsinger and Jansen 1993). Comparing DNAs from organisms belonging to different, even closely related taxa became as simple as comparing their restriction profiles. Indeed, a single nucleotide variation occurring at a given restriction cutting site is sufficient to affect cleavage and result in a different digestion pattern. A breakthrough in physical mapping of DNA by restriction enzyme digestion came about in 1976 with the introduction of the Southern blot approach. In essence, the experimental scenario was standardized by Edwin Southern's method to analyze specific DNA restriction fragments after sorting them electrophoretically by size through an agarose gel slab (Southern 1975). The possibility to analyze only a subset of fragments was of particular interest: the DNA was denatured within the gel at the end of the electrophoretic separation and the entire gel content was then transferred (blotted) onto a flexible membrane (usually a nitrocellulose filter) so as to make the DNA fragments bind to this support without altering the relative positions they had reached in the gel. The filter was then subjected to molecular hybridization with specific, radioactively labeled DNA probes. Thus, only the fragment(s) corresponding to the probe were evidenced.

A particularly interesting application of restriction mapping is based on the analysis of restriction fragment-length polymorphisms (RFLP). This technique allows to compare DNA samples drawn from different sources (individuals) by evaluating the size variability of specific restriction fragments. Fragments from a given DNA region of a single individual are compared with a reference sample or scored for an individual signature. RFLP analysis has gained wide acceptance as a highly accurate tool in prenatal diagnostics. The use of specific probes against single copy sequences, capable to distinguish sequence polymorphisms when hybridized to restriction endonuclease digested DNAs, has been proposed for the first time during the 1980s (Botstein et al. 1980; Weatherall et al. 1985). Later, this approach was also widely adopted by forensic molecular genetics (Sajantila and Budowle 1991; Balazs 1992). Nowadays conventional forensic serology has been almost completely replaced by DNA-based assays.

\section{Basic use of restriction enzymes: DNA manipulation}

The many heuristic and applicative approaches employing restriction enzymes have proved fundamental for physical DNA mapping. Similarly, recombinant DNA technology, which has equally strong ties with these extraordinary molecular tools, had a revolutionary impact on molecular biology as well as on biomedicine and biotechnology. Shortly before the identification of the first restriction enzymes, Lederberg (1952) proposed to use the term 'plasmid' for any extrachromosomal element determining heredity or sex. A few years later (Hickson et al. 1967) the physical and chemical properties of plasmid DNA and its circular nature were extensively characterized and plasmids were also visualized by electron microscopy. In 1972 Cohen and coworkers inserted an exogenous closed-circular DNA harboring sequences encoding the resistance against a given antibiotic into a bacterial strain. They selected the plasmidcontaining population by screening for the ability to grow in the presence of the same antibiotic (Cohen et al. 1972).

At that time, the capacity of DNA ligase to join two adjacent nucleotides aligned on a complementary template by creating a new phosphodiester bond had been demonstrated following experiments with E. coli extracts capable to join polydeoxynucleotide chains and convert hydrogen bonded circles from $\lambda$-phage into a covalently closed-circular form (Cozzarelli et al. 1967; Gefter et al. 1967; Gellert 1967; Olivera and Lehman 1967; Weiss and Richardson 1967). However, at the beginning of the 1970 s, a tool for the 
specific fragmentation of DNA was still missing. Stanley Cohen, one of the major personalities in the field, had been experimenting with mechanical DNA fragmentation (Cohen et al. 1967) but the right kind of highly specific 'molecular scissors' became available only through the studies of Arber, Smith and Nathans (Arber 1965; Smith and Wilcox 1970; Danna and Nathans 1971). Work from Herbert Boyer's lab represented a landmark by providing an historical restriction enzyme, EcoRI (Yoshimori et al. 1972). It also became clear that some class II activities produced a staggered cut in the double helix (figure 1), leaving complementary (the socalled sticky or cohesive) ends (Hershey et al. 1963; Gellert 1967), which could be bridged, regardless of the origin of the DNA, with DNA ligase (Jensen et al. 1971; Jackson et al. 1972; Lobban and Kaiser 1973). Suddenly, the recombinant DNA playground was ready. An excellent description about the origins and the development of molecular cloning, including an account of the famous meeting at Waikiki beach (Hawaii), has been published in recent years by Stanley Cohen (Cohen 2013).

By joining DNA fragments from different organisms, the generation of the so-called chimeric DNAs became possible. The insertion of some $X$. laevis rDNA fragments into the pSC101 plasmid was one of the first examples (Morrow et al. 1974). These experiments proved that it was possible to use bacterial plasmids to clone DNA from various sources; that the junction of DNAs from different organisms could take place after cutting them with restriction enzymes generating the same type of ends; and, last but not least, that this procedure did not affect the functionality of the plasmid itself which continued replicating and transcribing the harbored genes.

Then, another important step followed: the creation of gene libraries (reviewed in Durmaz et al. 2015). These libraries involved genomic DNA fragmentation by digestion with restriction enzymes cutting with high frequency, i.e. having short recognition sequences (4-6 bases). The fragments obtained were joined in vitro to a number of plasmid molecules ensuring statistically sufficient coverage of the whole genome. Thus, restriction fragments obtained from a given genome were distributed on different plasmids which collectively represented the whole genome of the organism. These libraries have allowed a systematic study of entire genomes, even those with a remarkably large size (e.g. the human one). Overall, the use of restriction enzymes to split large DNA chunks into fragments of defined size and with specific ends has paved the way not only to recombinant DNA technology but also and to the first DNA sequencing efforts (reviewed in Heather and Chain 2016).

Restriction enzyme-mediated manipulation of DNA has opened the possibility to introduce targeted deletions of gene or promoter sub-regions, in order to compare the behavior of deleted templates with wild-type copies in terms of substrates for RNA transcription/processing and translation. The ability to cut and join gene pieces almost at will has provided tremendous momentum to basic knowledge on the nature, function and regulation of genes, and has led to remarkable biotechnological achievements. It became possible to deeply engineer genes in vitro, even human ones, transcribe them and give rise, by subsequent translation, to proteins of medical interest such as globins or insulin. The latter was produced for the first time in 1979 (Goeddel et al. 1979). Since then, the production of complex, biologically active molecules by means of recombinant DNA technologies has become common practice (Khan et al. 2016).

These findings have greatly stimulated the research on site-specific manipulation of the genome, with emphasis on the development of endonuclease-based tools able to target and cleave virtually any sequence. This has led to two powerful systems: ZFN (zinc-finger nuclease) (Liu et al. 1997) and TALEN (transcription activator-like effector nuclease) (Christian et al. 2010; Miller et al. 2011). ZFN relies on artificial nucleases where the DNA-cleavage domain of Fok I, a type II restriction enzyme, is fused to the C-terminal of a zinc-finger DNA binding domain: in the fusion protein the Fok I nuclease domain is responsible for the cutting activity, whereas the zinc-finger domain recognizes and binds the target sequence. The TALEN system also exploits a fusion protein, constituted by the Fok I cleavage domain and a transcription activator-like effector DNAbinding domain (Gaj et al. 2013). Until 2011 ZFN and TALEN were the most promising systems endowed with high targeting/cutting specificity. However, in terms of time and economical requirements both systems have proven rather demanding and a substantial progress occurred with the introduction of programmable RNA-mediated targeting/cutting systems (Kim and Kim 2014) as RGEN (RNAguided engineered nucleases), which has been rapidly replaced by its more effective, and now widely adopted successor, CRISPR-Cas9 (see Section 6).

\section{DNA accessibility studies: chromatin, DNA methylation}

The study of chromatin structure has been largely based on nuclease resistance analyses (Hewish and Burgoyne 1973; Rill and Van Holde 1973; Noll 1974) that led to the basic nucleosome model proposed by R. Kornberg (Kornberg 1974). The nuclease approach was followed by another methodology (REAA, restriction enzyme accessibility assay) based on the cutting specificity of restriction enzymes to test the in vivo accessibility of specific DNA regions (Pfeiffer et al. 1975; Hörz et al. 1976; Lipchitz and Axel 1976). Successive reports showed that transcribed genes were more sensitive to restriction enzymes than non-transcribed ones (Grummt and Gross 1980). Cutting was hindered when the restriction site mapped within a DNA region associated with histones to form a nucleosome. Conversely, if the location of the nucleosome did not encompass the restriction site, cutting could occur. Thus, it became possible to observe nucleosome positioning or chromatin remodeling events 



Figure 1. Diagram showing the general mechanism of action of type II restriction endonucleases and the end products of the reaction. In the presence of $\mathrm{Mg}^{2+}$ ions a type II enzyme catalyzes the cleavage of phosphodiester bonds (solid arrows pointing at the bond between $3^{\prime} \mathrm{O}$ and P) at specific locations along the DNA, generating fragments with $5^{\prime}$-phosphoryl $/ 3^{\prime}$-hydroxyl ends. The reaction occurs by nucleophilic attack at the phosphorus atom, but it is as yet not fully established whether it proceeds by direct hydrolysis or through the formation of a covalent reaction intermediate (Pingoud et al. 2005). While individual chemical steps of the actual mechanism may exhibit specific variations for each restriction enzyme, the general scheme reported here is well conserved. The nucleotide sequence shown (GAATTC) is the recognition/cleavage site for EcoRI, a restriction enzyme which operates a staggered cut leaving $5^{\prime}$-protruding, cohesive ends.

mediated, for instance, by environmental changes. REAA has been used for a long time to evaluate changes in chromatin structure.

In 1978 Edwin Southern and Adrian Bird developed a mapping strategy involving the DNA methylation status (Bird and Southern 1978). In Xenopus laevis, ribosomal DNA from erythrocytes (somatic rDNA) showed higher resistance to the action of specific restriction enzymes as compared to the same substrate abundantly present in oocytes as amplified rDNA. The difference turned out to depend on the differential methylation status of the rDNA in the two tissues rather than on different nucleotide sequences. This approach gave rise to a number of comparisons exploiting differential methylation sensitivity to restriction enzymes and represents the origin of molecular epigenetics, which subsequently gained enormous impulse with the advent of high yield DNA sequencing (Lin 2018).

\section{CRISPR-Cas9: new frontiers in DNA editing}

The ability of restriction endonucleases to distinguish between methylated and non-methylated DNA substrates, is essential in safeguarding the bacterial genome against bacteriophage invasion. Restriction enzymes are regarded as a pivotal component of an innate defense system developed by 


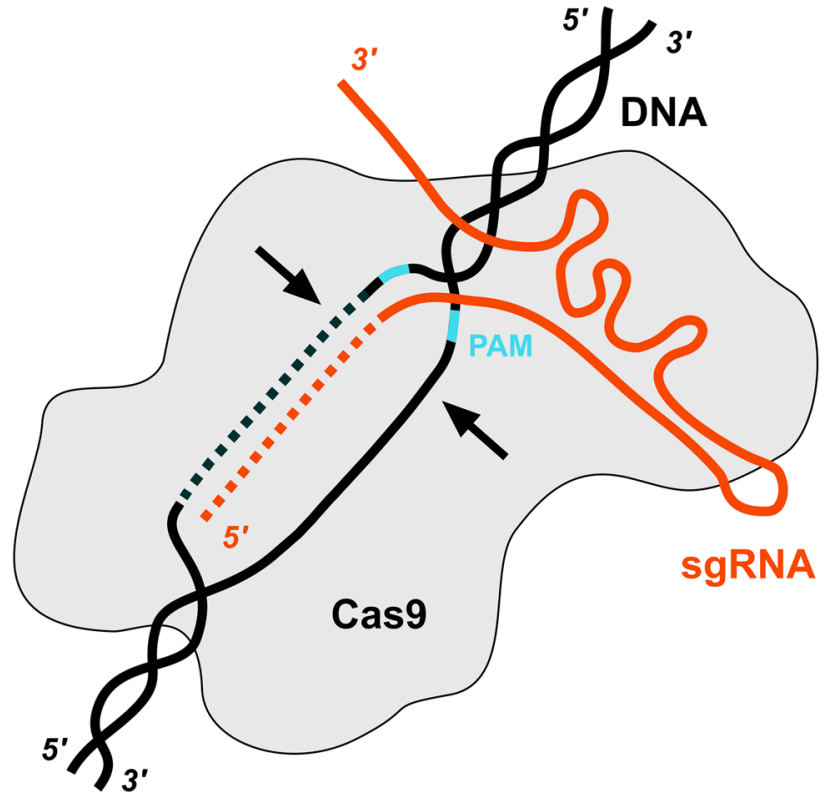

Figure 2. Simplified diagram of CRISPR-Cas9 as an RNAdriven DNA targeting/cleavage system. A synthetic small, singleguide RNA (sgRNA) carries a short sequence (usually 20 nucleotides; gray dotted line) able to match with a target DNA site (black dotted line). Target recognition is facilitated by the presence of a short sequence $\left(5^{\prime}\right.$-NGG), the protospacer-adjacent motif (PAM). Upon complementary base paring and R-loop formation the Cas9 endonuclease is activated and scission (arrows) of both DNA strands occurs (see Jiang and Doudna 2017 for a recent in-depth review). bacteria during evolution. An additional defense mechanism exploited by bacteria has recently stirred great interest. It is based on CRISPR elements (clustered regularly interspected short palindromic repeats) and on their associated proteins, Cas. At each phage infection, fragments from the phage genome are integrated into the bacterial genome at CRISPR loci (Barrangou et al. 2007). These loci are then transcribed and small RNAs originating from the integrated phage DNA fragments recognize the exogenous sequences. These RNAs are used as a guide by a specific Cas protein, Cas9 (CRISPR associated protein 9), an endonuclease encoded by a gene located near the CRISPR loci. When the RNA-guide pairs with the phage DNA sequence, Cas9 is activated and degrades phage DNA.

Both defense mechanisms, restriction/modification and CRISPR-Cas9, exploit endonucleolytic activities. The effectiveness of the first mechanism relies on the ability of the restriction endonuclease to distinguish and selectively inactivate phage DNA, while preserving host DNA, on the basis of the methylation status. The second mechanism (figure 2) takes advantage of the RNA guide forming a RNA/DNA duplex with phage DNA, and thus inducing the Cas9-mediated cleavage of the targeted sequence (this can occur even later, during a subsequent infection by the same phage).

Similarly to restriction enzymes, albeit at a much quicker pace, also the CRISPR-Cas9 system has undergone extensive studies to clarify the underlying molecular mechanisms and appropriately engineer them to manipulate DNA
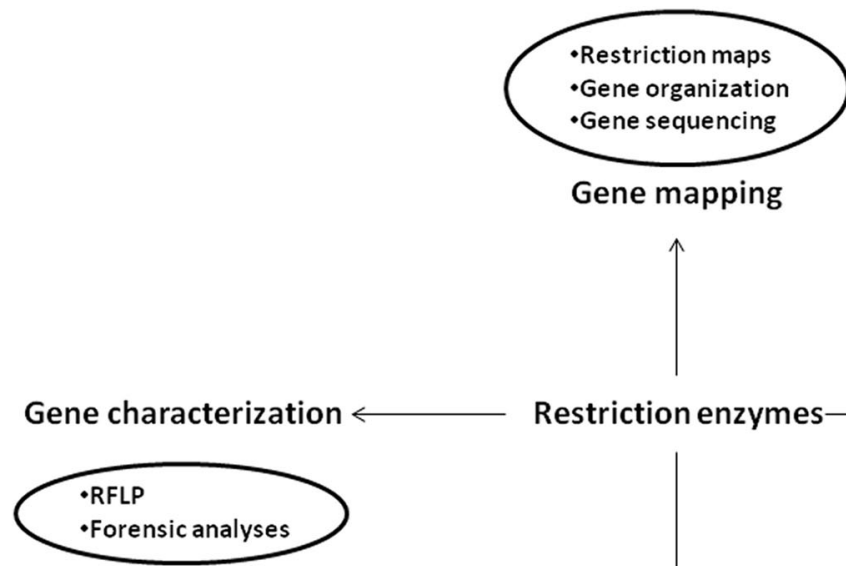

Restriction enzymes-

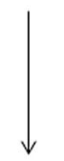

Gene cloning

-Deletion analyses

Gene accessibility

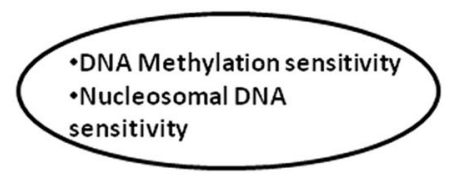

Restriction enzymes: active witness of DNA history

Figure 3. Restriction enzymes: active witnesses of DNA history. 


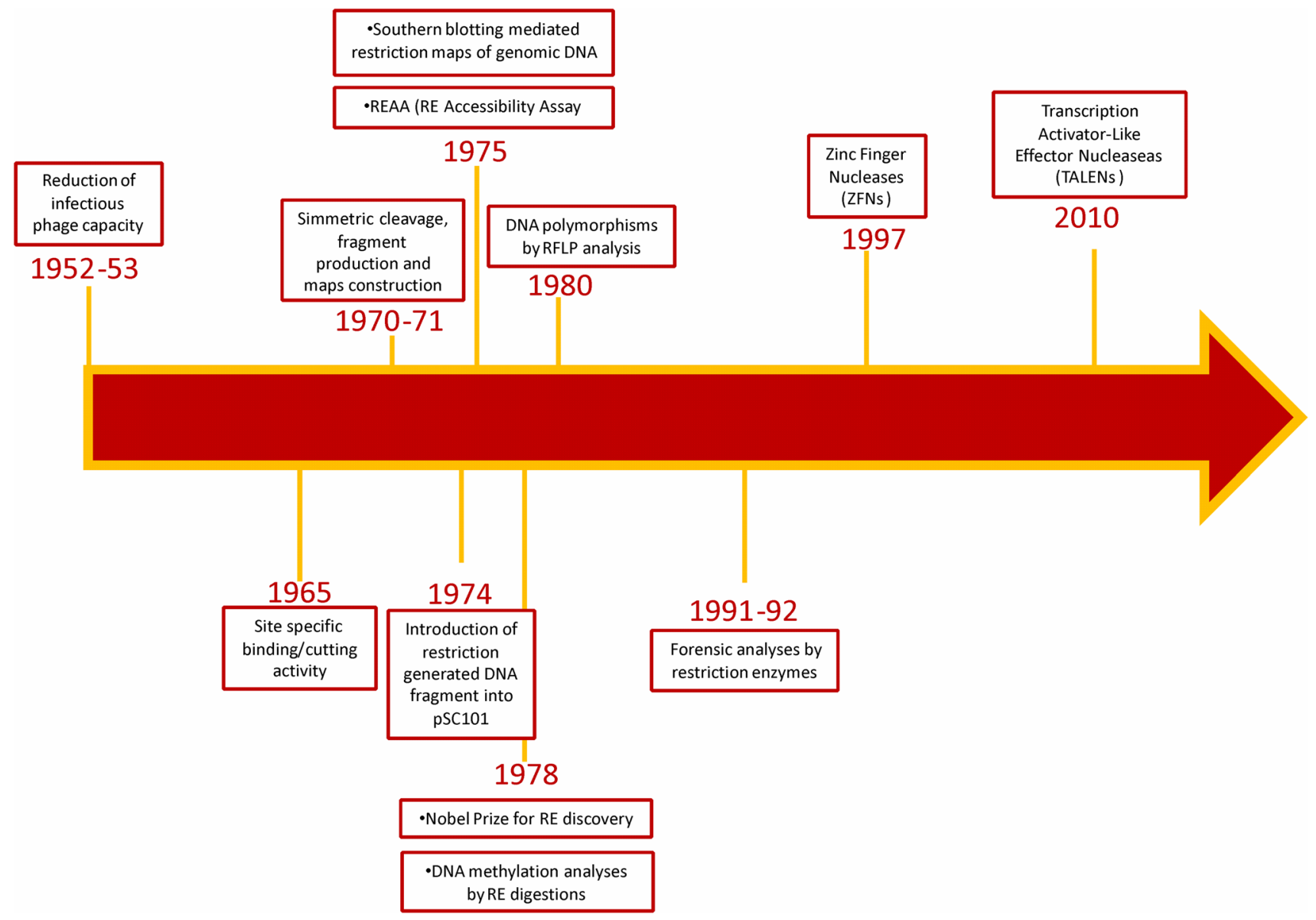

Figure 4. Timeline showing major milestones in the history of restriction enzymes.

templates. In essence, the CRISPR-Cas9 system has quickly turned into a novel and very powerful biotechnological tool, making genome editing virtually possible in any cell. This is exemplified by the possibility to introduce genetic mutations at specific locations by simply identifying an appropriate/ unique target DNA sequence and adopting the corresponding RNA guide to bring the Cas9 endonuclease in position (Jinek et al. 2012). Such a high specificity would not be achievable with restriction endonucleases as their recognition/cutting sites are dispersed all over the genome. Cutting sites of type II restriction enzymes are 'hard wired' into the structure of the protein, i.e. changing the recognition/cutting specificity requires a new protein. Conversely, the Cas9 endonuclease relies on a small guide RNA to pair with its complementary DNA target and a new guide RNA is sufficient to change the cutting specificity, without the need to modify the protein.

\section{Concluding remarks}

Since their discovery restriction enzymes have been widely used in fundamental DNA technology approaches like DNA cloning, mapping and manipulation. The availability of a molecular tool able to generate specific DNA fragments has been a turning point in the history of DNA. The involvement of restriction enzymes in a wide variety of sophisticated procedures makes these proteins active 'molecular witnesses' of the progresses in the analysis, manipulation and exploitation of the double helix (figure 3). What was initially a discovery driven by the interests and principles of basic research has rapidly become a powerful tool for applied science and translational approaches (figure 4). Interestingly, the CRISP/Cas system is currently following a closely similar path. Overall, these observations constitute an excellent exemplification of how basic, non-oriented research represents a very valuable reservoir of new discoveries with a strong applicative potential.

\section{References}

Arber W 1965 Host-controlled modification of bacteriophage. Annu. Rev. Microbiol. 19 365-378

Balazs I 1992 Forensic applications. Curr. Opin. Biotechnol. 3 18-23 
Barrangou R, Fremaux C, Deveau H, Richards M, Boyaval P, Moineau S, Romero DA and Horvath P 2007 CRISPR provides acquired resistance against viruses in prokaryotes. Science $\mathbf{3 1 5}$ 1709-1712

Bertani G and Weigle JJ 1953 Host controlled variation in bacterial viruses. J. Bacteriol. 65 113-121

Bird AP and Southern EM 1978 Use of restriction enzymes to study eukaryotic DNA methylation: I. The methylation pattern in ribosomal DNA from Xenopus laevis. J. Mol. Biol. 118 27-47

Botstein D, White RL, Skolnick M and Davis RW 1980 Construction of a genetic linkage map in man using restriction fragment length polymorphisms. Am. J. Hum. Genet. 32 314-331

Christian M, Cermak T, Doyle EL, Schmidt C, Zhang F, Hummel A, Bogdanove AJ and Voytas DF 2010 Targeting DNA doublestrand breaks with TAL effector nucleases. Genetics $\mathbf{1 8 6}$ 757-761

Cohen SN 2013 DNA cloning: a personal view after 40 years. PNAS 110 15521-15529

Cohen SN, Maitra U and Hurwitz J 1967 Role of DNA in RNA synthesis: XI. Selective transcription of $\lambda$ DNA segments in vitro by RNA polymerase of Escherichia coli. J. Mol. Biol. 26 19-38

Cohen SN, Chang ACY and Hsu L 1972 Nonchromosomal antibiotic resistance in bacteria: Genetic transformation of Escherichia coli by R-factor DNA*. Proc. Natl. Acad. Sci. USA 69 2110-2114

Cozzarelli NR, Melechen NE, Jovin TM and Kornberg A 1967 Polynucleotide cellulose as a substrate for a polynucleotide ligase induced by phage T4. Biochem. Biophys. Res. Commun. 28 578-586

Danna K and Nathans D 1971 Specific cleavage of Simian virus 40 DNA by restriction endonuclease of Hemophilus influenzae. Proc. Natl. Acad. Sci. USA 5 75-81

Durmaz AA, Karaca E, Demkow U, Toruner G, Schoumans J and Cogulu O 2015 Evolution of genetic techniques: Past, present, and beyond [WWW document]. BioMed Res. Int. https://doi. org/10.1155/2015/461524

Gaj T, Gersbach CA and Barbas CF 2013 ZFN, TALEN, and CRISPR/Cas-based methods for genome engineering. Trends Biotechnol. 31 397-405

Gefter ML, Becker A and Hurwitz J 1967 The enzymatic repair of DNA. I. Formation of circular lambda-DNA. Proc. Natl. Acad. Sci. USA 58 240-247

Gellert M 1967 Formation of covalent circles of lambda DNA by E. coli extracts. Proc. Natl. Acad. Sci. USA 57 148-155

Goeddel DV, Kleid DG, Bolivar F, Heyneker HL, Yansura DG, Crea R, Hirose T, Kraszewski A, Itakura K and Riggs AD 1979 Expression in Escherichia coli of chemically synthesized genes for human insulin. Proc. Natl. Acad. Sci. USA 76 106-110

Grummt I and Gross HJ 1980 Structural organization of mouse rDNA: comparison of transcribed and non-transcribed regions. Mol. Gen. Genet. 177 223-229

Gupta R, Capalash N and Sharma P 2012 Restriction endonucleases: natural and directed evolution. Appl. Microbiol. Biotechnol. 94 583-599

Heather JM and Chain B 2016 The sequence of sequencers: The history of sequencing DNA. Genomics 107 1-8

Hershey AD, Burgi E and Ingraham L 1963 Cohesion of DNA molecules isolated from phage lambda. Proc. Natl. Acad. Sci. USA 49 748-755
Hewish DR and Burgoyne LA 1973 Chromatin sub-structure. The digestion of chromatin DNA at regularly spaced sites by a nuclear deoxyribonuclease. Biochem. Biophys. Res. Commun. 52 504-510

Hickson FT, Roth TF and Helinski DR 1967 Circular DNA forms of a bacterial sex factor. Proc. Natl. Acad. Sci. USA 58 $1731-1738$

Holsinger KE and Jansen RK 1993 Phylogenetic analysis of restriction site data; in: Methods in enzymology, molecular evolution: Producing the biochemical data (eds) Zimmer EA, White TJ, Cann RL, Wilson AC (Cambridge, USA: Academic Press) pp 439-455

Hörz W, Igo-Kemenes T, Pfeiffer W and Zachau HG 1976 Specific cleavage of chromatin by restriction nucleases. Nucleic Acids Res. 3 3213-3226

Jackson DA, Symons RH and Berg P 1972 Biochemical method for inserting New genetic information into DNA of Simian virus 40: Circular SV40 DNA molecules containing lambda phage genes and the galactose operon of Escherichia coli. Proc. Natl. Acad. Sci. USA $692904-2909$

Jensen RH, Wodzinski RJ and Rogoff MH 1971 Enzymatic addition of cohesive ends to T7 DNA. Biochem. Biophys. Res. Commun. 43 384-392

Jiang F and Doudna JA 2017 CRISPR-cas9 structures and mechanisms. Annu. Rev. Biophys. 46 505-529

Jinek M, Chylinski K, Fonfara I, Hauer M, Doudna JA and Charpentier E 2012 A programmable dual-RNA-guided DNA endonuclease in adaptive bacterial immunity. Science 337 816-821

Kelly TJ and Smith HO 1970 A restriction enzyme from Hemophilus influenzae. II. J. Mol. Biol. 51 393-409

Khan S, Ullah MW, Siddique R, Nabi G, Manan S, Yousaf M and Hou H 2016 Role of recombinant DNA technology to improve life. Int. J. Genomics 2016 1-14

Kim H and Kim J-S 2014 A guide to genome engineering with programmable nucleases. Nat. Rev. Genet. 15 321-334

Knizewski L, Kinch LN, Grishin NV, Rychlewski L and Ginalski K 2007 Realm of PD-(D/E)XK nuclease superfamily revisited: detection of novel families with modified transitive meta profile searches. BMC Struct. Biol. 740

Kornberg RD 1974 Chromatin structure: a repeating unit of histones and DNA. Science 184 868-871

Lederberg J 1952 Cell genetics and hereditary symbiosis. Physiol. Rev. 32 403-430

Lin I-H 2018 Whole genome DNA methylation analysis using next-generation sequencing (BS-seq). Methods Mol. Biol. 1667 223-287

Lipchitz L and Axel R 1976 Restriction endonuclease cleavage of satellite DNA in intact bovine nuclei. Cell 9 355-364

Liu Q, Segal DJ, Ghiara JB and Barbas CF 1997 Design of polydactyl zinc-finger proteins for unique addressing within complex genomes. PNAS 94 5525-5530

Lobban PE and Kaiser AD 1973 Enzymatic end-to-end joining of DNA molecules. J. Mol. Biol. 78 453-471

Luria SE and Human ML 1952 A nonhereditary, host-induced variation of bacterial viruses. J. Bacteriol. 64 557-569

Miller JC, Tan S, Qiao G, Barlow KA, Wang J, Xia DF, Meng X, Paschon DE, Leung E, Hinkley SJ, Dulay GP, Hua KL, Ankoudinova I, Cost GJ, Urnov FD, Zhang HS, Holmes MC, Zhang L, Gregory PD and Rebar EJ 2011 A TALE nuclease 
architecture for efficient genome editing. Nat. Biotechnol. 29 143-148

Morrow JF, Cohen SN, Chang ACY, Boyer HW, Goodman HM and Helling RB 1974 Replication and transcription of eukaryotic DNA in Esherichia coli. Proc. Natl. Acad. Sci. USA 71 1743-1747

Noll M 1974 Subunit structure of chromatin. Nature 251 249-251

Olivera BM and Lehman IR 1967 Linkage of polynucleotides through phosphodiester bonds by an enzyme from Escherichia coli. Proc. Natl. Acad. Sci. USA 57 1426-1433

Pfeiffer W, Horz W, Igo-Kemenes T and Zachau HG 1975 Restriction nucleases as probes of chromatin structure. Nature $258450-452$

Pingoud A, Fuxreiter M, Pingoud V and Wende W 2005 Type II restriction endonucleases: Structure and mechanism. Cell. Mol. Life Sci. 62 685-707

Rill R and Van Holde KE 1973 Properties of nuclease-resistant fragments of calf thymus chromatin. J. Biol. Chem. 248 $1080-1083$

Roberts RJ, Belfort M, Bestor T, Bhagwat AS, Bickle TA, Bitinaite J, Blumenthal, RM, Degtyarev SK, et al. 2003 A nomenclature for restriction enzymes, DNA methyltransferases, homing endonucleases and their genes. Nucleic Acids Res. 31 1805-1812
Sajantila A and Budowle B 1991 Identification of individuals with DNA testing. Ann. Med. 23 637-642

Smith HO and Wilcox KW 1970 A restriction enzyme from Hemophilus influenzae. I. Purification and general properties. J. Mol. Biol. 51 379-391

Southern EM 1975 Detection of specific sequences among DNA fragments separated by gel electrophoresis. J. Mol. Biol. 98 503-517

Weatherall DJ, Old JM, Thein SL, Wainscoat JS and Clegg JB 1985 Prenatal diagnosis of the common haemoglobin disorders. $J$. Med. Genet. 22 422-430

Weiss B and Richardson CC 1967 Enzymatic breakage and joining of deoxyribonucleic acid, I. Repair of single-strand breaks in DNA by an enzyme system from Escherichia coli infected with T4 bacteriophage. Proc. Natl. Acad. Sci. USA 57 $1021-1028$

Williams RJ 2003 Restriction endonucleases: Classification, properties, and applications. Mol. Biotechnol. 23 225-243

Wilson GG and Murray NE 1991 Restriction and modification systems. Annu. Rev. Genet. 25 585-627

Yoshimori R, Roulland-Dussoix D and Boyer HW 1972 R factorcontrolled restriction and modification of deoxyribonucleic acid: Restriction mutants. J. Bacteriol. 112 1275-1279

Corresponding editor: SudHA BHATTACHARYA 